\title{
Pièces osseuses gravées du Solutréen moyen de la grotte Rochefort (Saint-Pierre-sur-Erve, Mayenne, France)
}

Stéphan Hinguant, Pierre-Elie Moullé, Almudena Arellano et Romain Pigeaud

\section{OpenEdition}

Journals

Édition électronique

URL : http://journals.openedition.org/paleo/2431

DOI : $10.4000 /$ paleo.2431

ISSN : $2101-0420$

Éditeur

SAMRA

Édition imprimée

Date de publication : 15 décembre 2012

Pagination : 337-356

ISSN : $1145-3370$

\section{Référence électronique}

Stéphan Hinguant, Pierre-Elie Moullé, Almudena Arellano et Romain Pigeaud, «Pièces osseuses gravées du Solutréen moyen de la grotte Rochefort (Saint-Pierre-sur-Erve, Mayenne, France) », PALEO [En ligne], 23 | 2012, mis en ligne le 29 avril 2013, consulté le 26 juillet 2020. URL : http:// journals.openedition.org/paleo/2431; DOI : https://doi.org/10.4000/paleo.2431

Ce document a été généré automatiquement le 26 juillet 2020.

\section{cc) (†)}

PALEO est mis à disposition selon les termes de la licence Creative Commons Attribution - Pas d'Utilisation Commerciale - Pas de Modification 4.0 International. 


\title{
Pièces osseuses gravées du Solutréen moyen de la grotte Rochefort (Saint-Pierre-sur-Erve, Mayenne, France)
}

\author{
Stéphan Hinguant, Pierre-Elie Moullé, Almudena Arellano et Romain \\ Pigeaud
}

\section{1 - Présentation du site}

1 La grotte Rochefort, sur la commune de Saint-Pierre-sur-Erve, est une cavité appartenant à l'ensemble des grottes dites de Saulges, toutes localisées le long de l'Erve sur environ 1,5 km de son parcours (fig. 1). Connue de longue date, fréquentée depuis le Paléolithique, elle est aujourd'hui une des deux grottes du site à être ouverte au public dans le cadre de visites guidées thématiques. Il s'agit d'une des cavités les plus vastes de l'ensemble, dont le développement, comprenant un réseau supérieur et un réseau inférieur, atteint $250 \mathrm{~m}$. Seul le premier a été accessible au cours des temps préhistoriques, comprenant un couloir d'accès long de $24 \mathrm{~m}$ et une grande salle dans laquelle il débouche. Malgré plusieurs fouilles à la fin du XIXe siècle et dans les années 1930, qui ont permis d'identifier une présence paléolithique dans la grotte, les surfaces disponibles pour des recherches archéologiques modernes demeurent importantes. Depuis 2001, une fouille pluriannuelle est engagée dans une partie de la grande salle, travaux à l'origine de l'identification d'un remarquable niveau solutréen (fig. 1). Tant sur le plan des assemblages lithiques et fauniques que par la présence d'un art sur plaquettes et sur os, la couche solutréenne de la grotte Rochefort présente en effet toutes les caractéristiques d'un véritable habitat (Hinguant et Colleter dir. 2010 ; Hinguant et Biard à paraître ; Pigeaud à paraître).

2 Pour des raisons pratiques, la zone fouillée ne concerne actuellement que la moitié de la salle. Les fouilles montrent clairement que nous nous trouvons en périphérie de 
l'occupation, dans un secteur ou les rejets semblent importants et où l'organisation spatiale n'est guère perceptible. Pour autant, un rejet de foyer et une structure aménagée avec des blocs de calcaire, en limite d'emprise de fouille, soulignent l'existence d'un sol d'occupation. Les raccords et remontages entre pièces lithiques ou osseuses montrent par ailleurs que des zones préférentielles d'activités existent. Malgré d'évidents problèmes post-dépositionnels, notamment liés à la cryoturbation et aux ruissellements, la lecture spatiale apporte donc de réelles informations, opportunément renforcées par la remarquable qualité de conservation des objets mobiliers.

3 Stratigraphie et datations radiocarbone placent l'occupation au cœur du Solutréen, entre 19000 et $20000 \mathrm{BP}$, chronologie confirmée par la culture matérielle ne comptant ni pointes à face plane ni pointes à cran mais où domine la feuille de laurier (fig. 2). Il faut souligner ici la remarquable homogénéité de cette unité solutréenne, excluant tout mélange possible avec des chronocultures antérieures et/ou postérieures. Faune et données paléoenvironnementales assurent quant à elles l'existence d'un environnement froid et sec, correspondant bien au Maximum Glaciaire (OIS 2), dans lequel subsistent néanmoins des zones protégées favorables au maintien d'espèces davantage tempérées. Se côtoient ainsi dans le corpus faunique, avec le renne et le cheval qui dominent le spectre, le bouquetin des Alpes, le bison, le rhinocéros laineux, le mammouth, le renard polaire, le loup, mais également l'ours brun, le lynx, le chat sylvestre ou encore le sanglier (Hinguant et Colleter dir. 2010 ; Hinguant et al. à paraître). L'unité solutréenne de la grotte, à ce stade des recherches, est d'une puissance maximale de 50 à $60 \mathrm{~cm}$, composée de clastes calcaires issus de l'érosion d'une cheminée de soutirage et de fines sablo-argileuses comblant les interstices. Six subdivisions sont enregistrées (4.1 à 4.6), correspondant à des changements de structures, de textures et de couleur dans la stratigraphie, mais aussi à des densités différentes de mobilier. Si quelques mélanges verticaux sont notés, l'homogénéité de chacune des strates semble préservée, notamment pour les subdivisions 4.2 et 4.3 , sans que l'on sache néanmoins si elles correspondent à la superposition d'occupations successives (saisonnières ou autres).

4 C'est au sein d'un corpus mobilier fort d'environ 8000 pièces, dont près de 5500 restes osseux, 2000 pièces lithiques taillées et 300 plaquettes gravées, que se comptent les objets exposés ici.

\section{2 - Corpus}

5 Les pièces proviennent indistinctement des sous-couches de l'unité 4 avec cependant une majorité en 4.2 et 4.3 . Un certain nombre a été collecté au tamisage et/ou simplement localisé par carré (NC pour non coté). La répartition spatiale des pièces cotées au sein de la zone fouillée n'apporte aucune information particulière si ce n'est une concentration plus importante d'objets dans la moitié nord de la fouille, constat valable pour la majorité des artefacts solutréens (fig. 3).

Remarquables par leur qualité de conservation, malgré une fragmentation fréquente, ces os gravés sont présentés dans un inventaire descriptif selon la nature et l'agencement des incisions. Les os portant une expression graphique incontestable sont évidemment mis en avant. Dix pièces sont inventoriées dans cette catégorie. D'autres os portent des incisions dont l'aspect et l'agencement régulier posent la question de leur 
nature ; graphisme ou simples traces de décarnisation ? C'est par exemple le cas de quelques pièces qui ont pu servir de billot mais elles portent des traces dont la régularité peut aussi être perçue comme volontaire, ce qui explique leur présentation dans ce corpus. Notons ici que de très nombreuses pièces osseuses de la couche solutréenne de la grotte Rochefort sont affectées de stigmates liés aux activités de boucherie (stries de découpe, raclage etc.). Si nous avons isolé ici quelques pièces, c'est donc avant tout parce que les stigmates repérés dénotent dans l'inventaire des habituelles traces, surtout compte tenu des supports évoqués, en l'occurrence majoritairement des côtes. N'oublions pas également les marques qu'ont pu laisser les rongeurs sur certains os même si, dans le corpus présenté, la confusion n'est pas possible. Enfin, la combinaison de plusieurs de ces stigmates sur une même pièce est parfois possible.

Figure 1 - Localisation géographique et topographique du site et plan de la grotte Rochefort montrant la répartition des vestiges mobiliers solutréens (infographie R. Colleter).

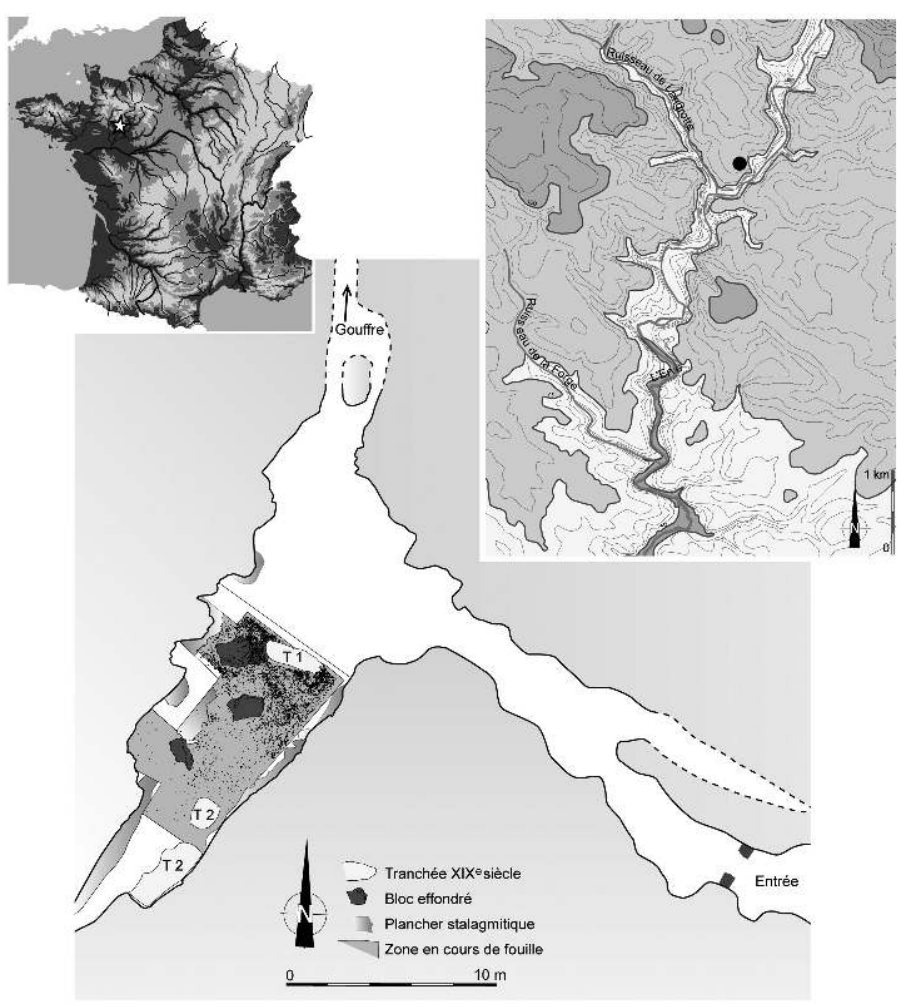

\section{1 - Os portant une expression graphique}


Figure 2 - Stratigraphie synthétique du remplissage supérieur de la grotte Rochefort et datations ${ }^{14} \mathrm{C}$ des niveaux solutréens (dessin S. Hinguant, photos et infographie R. Colleter)

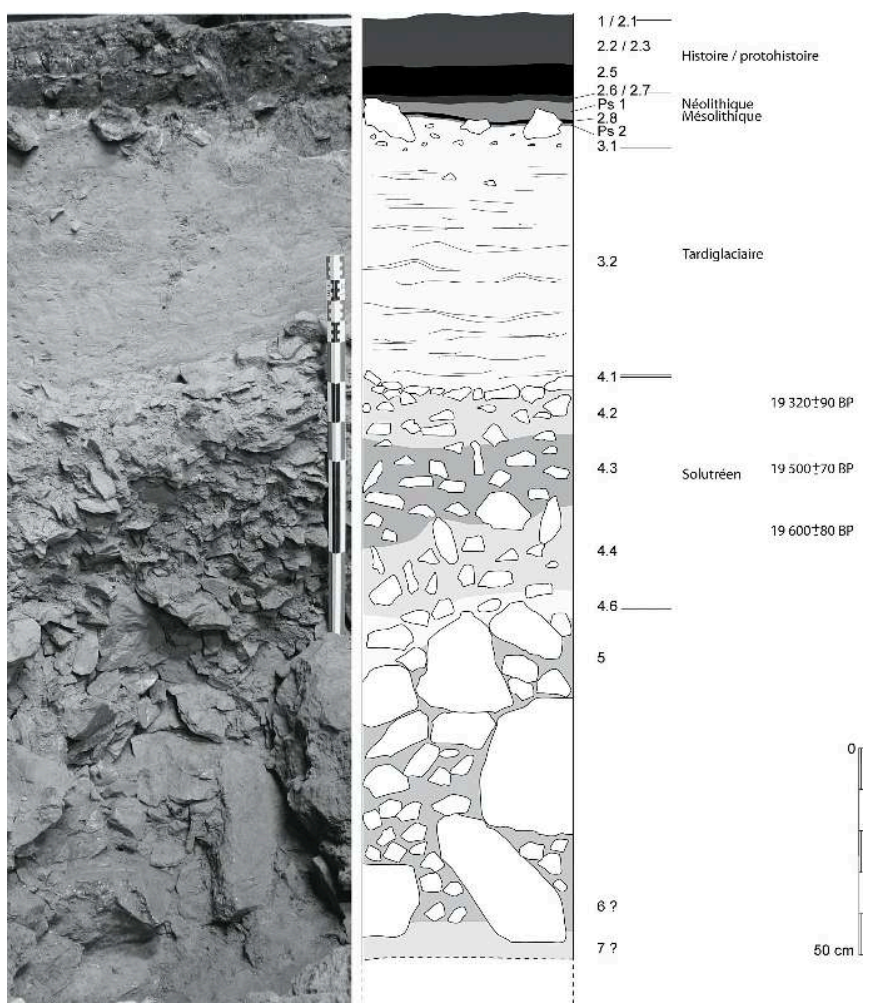

Figure 3 - Carte de répartition des côtes, os et défense gravés du Solutréen de la grotte Rochefort (infographie R. Colleter).

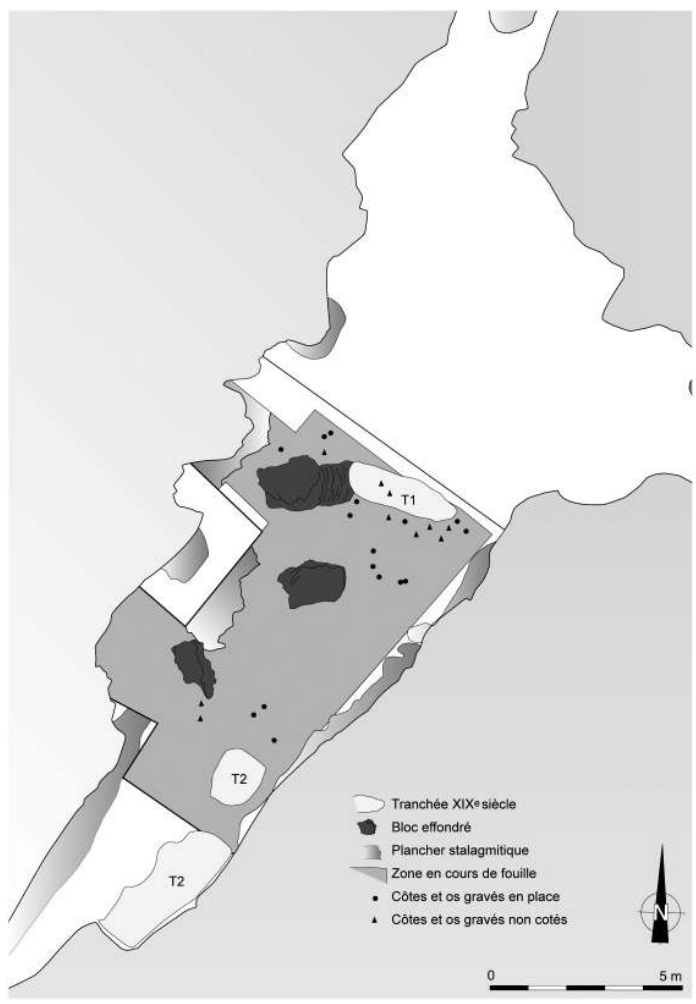


Figure 4 - Diaphyses ou fragments diaphysaires de côtes d'herbivores gravées (dessins S. Hinguant sauf $n^{\circ} 2, N$. Mélard, photos P.-É. Moullé et R. Colleter, infographie R. Colleter).

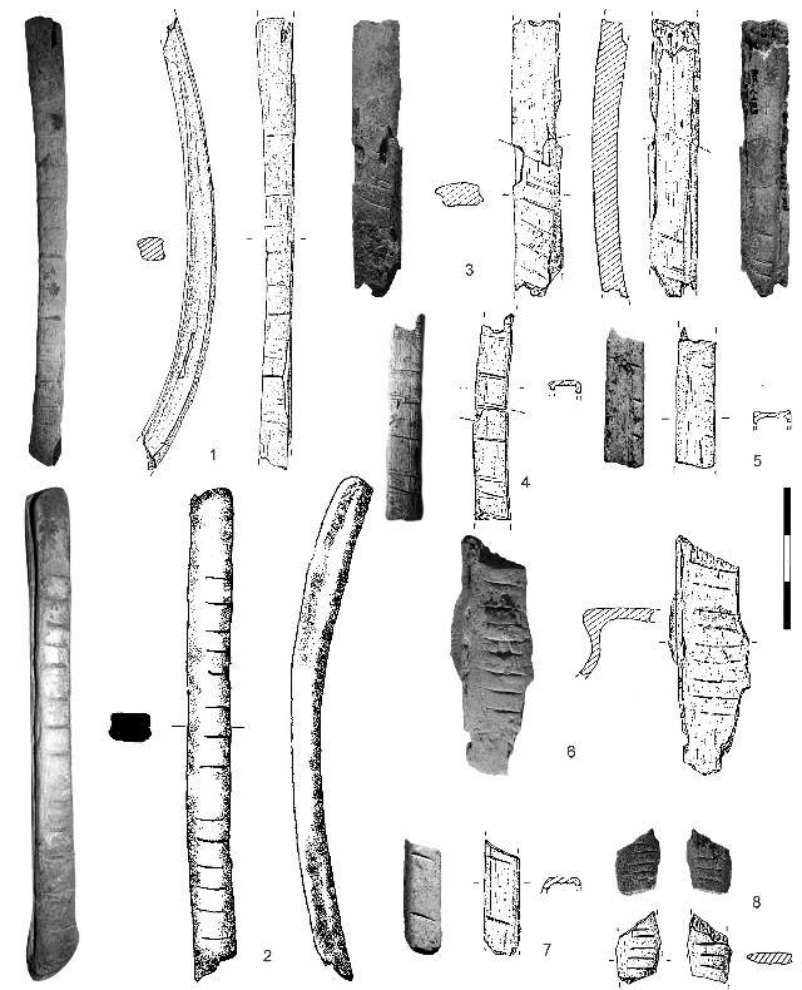

- $\mathbf{n}^{\circ} 2951$ (4.3) : fragment de côte de petit herbivore (bouquetin ?) pouvant correspondre à une dixième côte gauche. Les extrémités dorsale et ventrale manquent. Présence d'au moins 12 stries perpendiculaires au grand axe, très régulières, évoquant la composition décorant la côte d'ours $\mathrm{n}^{\circ} 984$ ( $\mathrm{cf}$ infra). Une treizième incision est presque entièrement cachée par de la concrétion et une quatorzième, moins longue et décalée vers le bord de la côte, est également visible. Par ailleurs, le bord caudal de l'extrémité dorsale porte une strie profonde qui a marqué les deux côtés saillants de l'os sans atteindre la concavité. L'origine symbolique ou décorative de ces marques nous semble ici évidente (fig. $4, \mathrm{n}^{\circ} 1$ ).

- $\mathbf{n}^{\circ} 242$ (musée de Dresde, Allemagne, coll. De Boxberg) : pièce issue des fouilles anciennes de De Boxberg, le musée Crozatier du Puy-en-Velay en possédant également un moulage (fig. 4, $\mathrm{n}^{\circ} 2$ ). Sa provenance exacte est inconnue mais son appartenance à l'unité solutréenne ne fait guère de doute (Monnier et al. 2005). Il s'agit d'un long fragment de côte de petit herbivore portant sur sa face externe une série de 14 incisions parallèles régulièrement espacées, composition évoquant plusieurs spécimens découverts in situ dans la grotte Rochefort dont la pièce précédemment décrite.

- $\mathbf{n}^{\circ} 2189$ (4.3) : fragment de côte de petit herbivore (bouquetin ?). Sur la face interne, des stries longues longitudinales correspondent à un raclage. Des stries perpendiculaires légèrement obliques recoupent ces stries longitudinales selon un rythme formant trois ensembles. Chaque ensemble est composé d'au moins deux stries fines très rapprochées témoignant de l'utilisation d'un outil de silex à la partie active double plutôt que de plusieurs passages sur un même tracé dans le but d'en accentuer le marquage. Sur la face externe, se distinguent deux groupes de stries perpendiculaires, légèrement obliques, selon le même modèle. Un simple travail de boucherie ne permet pas d'expliquer ces compositions (fig. $4, \mathrm{n}^{\circ} 3$ ). 
- $\mathbf{n}^{\circ} 1837$ (4.2) : fragment de côte de petit herbivore avec seulement la face supérieure conservée. Celle-ci présente au moins six stries ou groupes de stries transversales espacées régulièrement qui ne peuvent être interprétées comme de simples stigmates de boucherie (fig. 4, $\mathrm{n}^{\circ} 4$ ).

- NC devant est $n^{\circ} 3$ (4.3) : fragment de côte (entre la 7è et la 10è) de petit herbivore (bouquetin ?). Il s'agit d'un fragment de côte crânial ou caudal provenant d'une zone proche de l'extrémité dorsale. Trois stries courtes à section en $\mathrm{V}$ régulièrement espacées sont présentes sur le fragment. Deux d'entre elles affectent les côtés saillants du bord sans atteindre la concavité entre ces bords tandis que la troisième strie ne marque qu'un côté saillant. La disposition de l'ensemble ne peut s'expliquer par un travail de boucherie (fig. $\left.4, n^{\circ} 5\right)$.

- $\mathbf{n}^{\circ} 3167$ (4.3) : fragment d'os non déterminé présentant un registre de neuf stries parallèles régulièrement espacées. À une extrémité du fragment, la cassure s'est produite en partie selon le tracé d'une strie, suggérant que le décor se poursuivait sur la pièce (fig. $4, \mathrm{n}^{\circ} 6$ ).

- NC devant est $n^{\circ} 5$ (4.3) : fragment de côte de petit herbivore avec seulement la face externe conservée. Celle-ci montre deux stries transversales légèrement obliques, bien marquées, à section en $\mathrm{V}$, qui ne peuvent pas correspondre à des stries de boucherie (fig. 4, $\left.\mathrm{n}^{\circ} 7\right)$.

- n⿳㇒ 4268 (4.4) : petit tronçon de côte d'un herbivore (?), sur lequel l'agencement, la régularité et la profondeur des incisions, sur les deux faces de l'os, sont particulièrement nets (fig. $4, \mathrm{n}^{\circ}$ $8)$.

- $\mathbf{n}^{\circ} 984$ (4.2) : découverte en 2006 dans la couche 4.2, c'est à ce jour la pièce maîtresse de la collection. L'objet est une côte droite d'ours brun (Ursus arctos), pratiquement complète (fig. 5). Vers l'extrémité dorsale, il manque le tubercule et la facette articulaire de ce dernier, le col et la tête. Il n'y a pas d'extrémité ventrale ; l'articulation costo-chondrale est donc absente. À l'extrémité dorsale du corps, sur le bord caudal, la tubérosité du muscle longissimus est bien conservée. Au même niveau mais sur la face interne, la tubérosité du muscle ilio-costal est altérée et partiellement couverte de concrétion. À la suite de ces tubérosités, le long du corps, l'angle de jonction entre le bord caudal et la face interne est abrasé jusqu'à faire apparaître la spongiosasur une longueur correspondant à la zone des incisions perpendiculaires d'origine anthropique. Cette abrasion peut être également d'origine anthropique comme l'indique en particulier son aspect poli. La présence de nombreuses stries longitudinales et obliques sur le corps peut par ailleurs faire penser à un nettoyage, une préparation de la côte. Davantage vers l'extrémité ventrale, les angles entre le bord caudal et les faces externe et interne présentent également des zones abrasées polies. L'angle de la côte (entre l'extrémité dorsale et le corps) est assez ouvert, ce qui exclut une position vers les premières côtes de la cage thoracique (14 paires chez l'ours brun). Ce qui est conservé du corps indique une côte assez longue qui peut correspondre à une position plutôt centrale (7e-9e position ?). Cette côte paraît appartenir à un individu de grande taille mais elle présente un aspect plus gracile que des exemplaires observés d'ours des cavernes. Rappelons que les couches 4.2 et 4.3 de la grotte Rochefort ont livré plusieurs restes d'ours brun de grande taille, certains portant des stigmates de boucherie. Cette côte a servi de support à quatorze incisions parallèles et profondes inscrites sur la face interne de l'os, plus proches de l'extrémité vertébrale que ventrale. Ces stries, par leur morphologie et leur régularité, comme par leur position sur l'os, ne sont visiblement pas en lien avec un comportement de boucherie, pratique rare mais déjà attestée sur ce type de reste (Armand et al. 2003 ; Quilès 2004 ; Armand 2006 ; Vercoutère et al. 2006). Nous sommes bien en présence d'un acte intentionnel à but décoratif ou symbolique, un objectif fonctionnel de 
type comptage ne pouvant pas être exclu même s'il est impossible à démontrer (Pernat 2001). L'observation de l'ensemble gravé permet d'identifier trois registres potentiels, pouvant traduire une chronologie dans l'exécution des stries, sans toutefois plus de détails. Les deux incisions les plus proches de l'extrémité dorsale sont très profondes et plus larges que les autres. L'espace entre les deux est par ailleurs réduit $(3 \mathrm{~mm})$. Les six incisions suivantes, demeurant profondes, sont systématiquement accompagnées de stries plus fines parallèles. Elles peuvent traduire des repentirs ou une insistance des gestes de l'artisan avant de trouver la position recherchée pour chaque encoche. Par ailleurs, l'équidistance entre les stries principales est ici régulière, avec un espacement de l'ordre de $8 \mathrm{~mm}$. Un dernier registre de six incisions, plus discrètes car moins longues et moins profondes que les précédentes, termine (ou débute ?) l'ensemble. L'équidistance entre chaque marque est là aussi très régulière $(6 \mathrm{~mm})$. Une observation attentive de la pièce permet de voir que la côte est finement raclée, voire polie, sur une grande partie de sa surface, notamment vers l'extrémité ventrale. Des traces de stries de découpe sont également encore visibles en partie médiane de la pièce, beaucoup plus fines et donc discernables de celles qui composent le « décor » proprement dit. Par ailleurs, on note la présence sporadique de voiles d'ocre rouge.

- $\mathbf{n}^{\circ} 8810$ (4.3) : fragment de défense de mammouth (Mammuthus primigenius). La pièce, rendue fragile par la nature même de l'ivoire se dégradant en " peau d'oignon ", a pu être consolidée. Après restauration, elle mesure $17 \mathrm{~cm}$ de longueur pour $3,5 \mathrm{~cm}$ de largeur moyenne et $5 \mathrm{~mm}$ d'épaisseur moyenne (fig. 6). Cette dernière mesure correspond bien à l'épaisseur régulière que présentent les cornets d'ivoire s'emboîtant les uns dans les autres dans une défense. Intimement liés entre eux du vivant de l'animal ou dans une défense encore " fraîche ", ces différents éléments se délitent avec le temps ce qui suggère que la pièce a probablement été collectée à l'état fossile (Poplin 1995). La restitution du diamètre moyen d'origine avoisine $7 \mathrm{~cm}$ et la morphologie comme la courbure, avec toutes les réserves nécessaires, semblent placer le fragment plutôt en partie distale de la défense. Comme sur les côtes d'herbivores présentées ci-dessus, la face externe porte une série d'incisions très fines, parallèles et équidistantes. Un total de 11 traits compose le motif mais certaines stries se doublent voire se triplent. Un examen sous microscope permet en effet de voir que les incisions ont été réalisées au burin, la courbure du support provoquant à plusieurs reprises un changement d'axe au moment de la réalisation du geste. Deux voire parfois trois fines incisions se relayent alors sur un même tracé (fig. $7 \mathrm{~b}$ et c). Il semble que la composition soit complète, la partie proximale conservée, malgré sa relative altération, ne montrant pas de stries. En partie distale, comme sur un des bords de la défense, les nombreuses striures et les grignotages provoqués par les rongeurs gênent la lecture de la pièce mais là aussi les quelques tracés visibles semblent s'arrêter avant le bord (fig. 7a). Des stries de longueur limitée affectent également la surface ornée selon le grand axe de la défense, ou ponctuellement, de manière désordonnée. Mais elles sont sans commune mesure avec les incisions composant le motif et ne doivent sans doute pas y participer. 
Figure 5 - Côte d'ours brun (Ursus arctos) n 984 gravée (dessin S. Hinguant, photos H. Paitier, infographie R. Colleter) (échelle réduite).
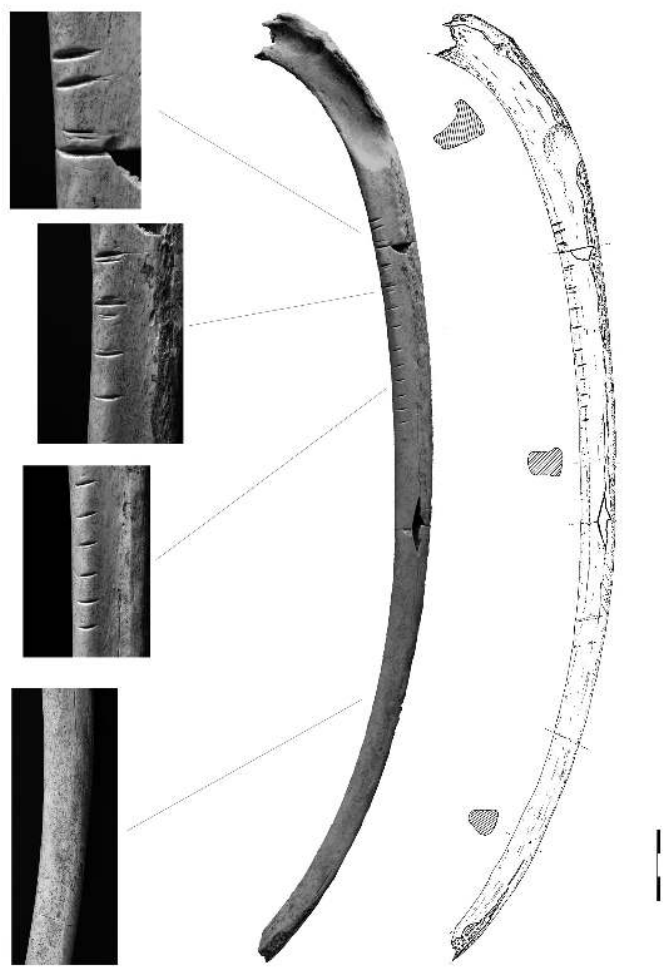

Figure 6 - Fragment de défense de mammouth (Mammuthus primigenius) incisée $n^{\circ} 8110$ (dessin $S$. Hinguant, photo et infographie R. Colleter).
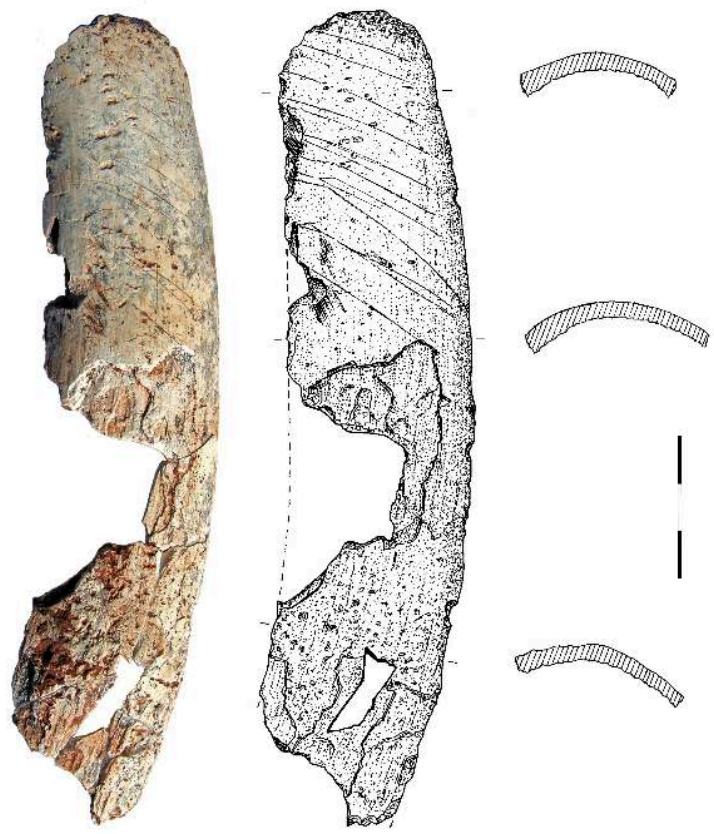
Figure 7 - Détail des incisions de la défense de mammouth incisée $n^{\circ} 8110$ a, b et $c$ : vues externes ; $d$ : vue interne. Agrandissements variés (photos et infographie R. Colleter).
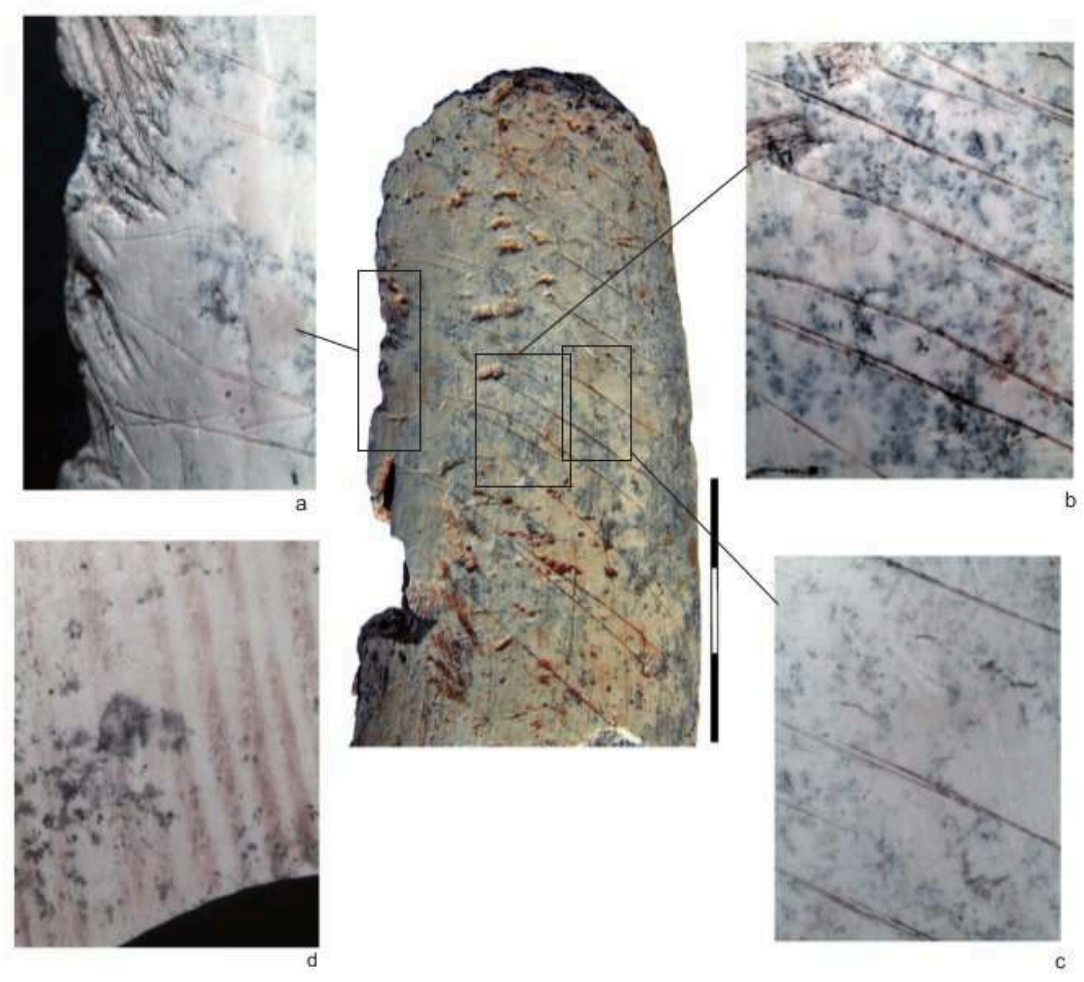

\section{2 - Os portant une expression graphique possible}

- $\mathbf{n}^{\circ} 1870$ (4.2) : long fragment de côte de petit herbivore (bouquetin ?). Présence de trois stries obliques très nettes sur la face interne, dont l'une à l'origine de la cassure qui, compte tenu de leur régularité, ne devraient pas correspondre à un travail de dépeçage (fig. $8, \mathrm{n}^{\circ} 1$ ). La fonction de stries d'adhérence n'est cependant pas exclue.

- $\mathbf{n}^{\circ} 1817$ (4.2) : fragment de côte d'herbivore (renne ou équidé ?). Présence d'au moins cinq stries obliques, parfois doublées, qui pourraient correspondre à un travail de boucherie (dépeçage) mais la régularité de l'espacement entre chaque incision est à noter (fig. $8, \mathrm{n}^{\circ} 2$ ).

- NC devant est $\mathbf{n}^{\circ} \mathbf{1}$ (4.3) : fragment de côte de petit herbivore. Sur sa face interne, trois stries perpendiculaires à section en $\mathrm{V}$ sont espacées d'un centimètre l'une de l'autre. La strie du milieu est accompagnée de deux autres incisions plus fines qui pourraient correspondre à des tentatives avortées de gravure ou à des repentirs dans la position. Ces stries ne semblent pas correspondre à un travail de boucherie (fig. $8, n^{\circ} 3$ ). Un raccord de cette pièce avec l'élément NC devant est $\mathrm{n}^{\circ} 5$, issu de la même couche (fig. $4, \mathrm{n}^{\circ} 7$ ), est d'ailleurs possible, ce qui placerait l'objet dans les pièces incontestablement gravées.

- NC devant est $\mathbf{n}^{\circ} \mathbf{2}$ (4.3) : fragment de côte de petit herbivore, avec sur la face interne deux stries larges et profondes en $\mathrm{V}$ accompagnées d'autres stries plus fines. Elles ne correspondent probablement pas à un travail de boucherie (fig. $8, \mathrm{n}^{\circ} 4$ ).

- $\mathbf{n}^{\circ} 6935$ (4.3) : fragment de côte ou os plat présentant sur sa face externe une série de 13 stries au minimum, plus ou moins parallèles et régulièrement espacées. Malgré la fragilité de la pièce et l'état squameux du cortex de l'os, les incisions sont encore nettes et profondes. Une pratique liée à la boucherie n'est cependant pas exclue dans ce cas (fig. 8, n 5). 
- NC fond ouest $\mathbf{n}^{\circ} \mathbf{1}(\mathbf{4 . 2 )}$ : petit fragment de côte, avec une seule face conservée qui présente une abrasion faisant apparaître des zones du tissu spongieux. Cette face présente une plage de stries larges en V, entrecroisées, pouvant appartenir à un motif figuratif (fig. 8, nº 6).

\section{3 - Os portant des traces de décarnisation ou ayant servi de billot}

- $\mathbf{n}^{\circ}$ 991/1087/1703 NC (4.2) : côte d'herbivore de taille moyenne, probablement une dixième côte gauche de bouquetin, découverte en quatre fragments séparés (fig. $9, \mathrm{n}^{\circ} 1 \mathrm{a}-\mathrm{c}$ ). Les fragments $n^{\circ} 1703 \mathrm{NC}(1 \mathrm{~b})$ et 991 (1c) sont proches de l'extrémité dorsale (courbure accentuée). Le premier présente sur sa face externe une vingtaine de stries fines et serrées, obliques par rapport au grand axe de l'os. Le fragment $n^{\circ} 991$ fait suite au précédent mais sans raccord direct. Il porte sur sa face externe deux séries de stries fines, droites (perpendiculaires au grand axe de l'os) ou obliques parallèles. Le fragment $n^{\circ} 1087$ (1a) est sans raccord direct avec le $n^{\circ} 1703$ NC. Il correspond à la partie ventrale de la côte et son extrémité est proche de l'articulation costo-chondrale. Ce fragment présente sur les faces interne et externe des stries longues parallèles au grand axe de la pièce. Des stries courtes obliques sont également présentes sur la face externe vers l'extrémité dorsale du fragment. Un travail de boucherie pourrait seul expliquer la présence de certaines de ces stries (stries fines, courtes, obliques ou perpendiculaires par rapport au grand axe de l'os) mais leur nombre important ainsi que les traces de raclage volontaire sur les faces externe et interne pourraient aussi permettre d'évoquer une préparation de l'os pour un tout autre usage.

- no 1757 (4.2) : fragment de diaphyse de métatarsien de renne (Rangifer tarandus) avec bord de fracture lisse et traces de brûlure. Des stries discrètes affectent la pièce avec une régularité d'espacement marquée (fig. $9, n^{\circ} 2$ ). Il n'est cependant pas exclu que ces marques soient naturelles.

- $\mathbf{n}^{\circ} 2304$ (4.3) : fragment de diaphyse (fémur de renne ou bouquetin ?) présentant des marques transversales. Au premier abord, ces marques font penser à une série de stries parallèles disposées intentionnellement de façon régulière. L'observation à la loupe montre qu'il ne s'agit pas de stries profondes à section en $\mathrm{V}$, mais de marques peu profondes et assez larges à section en U. Il peut donc s'agir de marques de dents ou de griffes. Outre cette série de marques parallèles, s'observent d'autres incisions de même aspect mais disposées de façon plus anarchique (fig. 9, $\mathrm{n}^{\circ} 3$ ).

- $\mathbf{n}^{\circ}$ 5203a (4.4) : fragment de côte indéterminée présentant au moins six marques subparallèles sur une de ses faces. Les stries ne sont cependant pas dans ce cas totalement incompatibles avec des gestes de boucherie (fig. 9, $\mathrm{n}^{\circ} 4$ ).

- NC $\mathbf{n}^{\circ} \mathbf{1}$ (4.2) : fragment de côte avec au moins sept stries fines courtes et obliques pouvant être de boucherie (fig. $9, \mathrm{n}^{\circ} 5$ ).

- NC devant ouest $n^{\circ} 1$ (4.3) : fragment d'os non déterminé présentant un groupe de trois stries fines (travail de boucherie ou utilisation de l'os ?) (fig. $9, \mathrm{n}^{\circ} 6$ ).

- NC devant est $n^{\circ} 4$ (4.3) : fragment d'os plat présentant des stries sub-parallèles obliques selon le grand axe du fragment, pouvant éventuellement correspondre à des stries de boucherie (fig. 9, $\mathrm{n}^{\circ} 7$ ). 
Figure 8 - Fragments de côtes ou os d'herbivores incisés (dessins S. Hinguant, photographie et infographie R. Colleter).

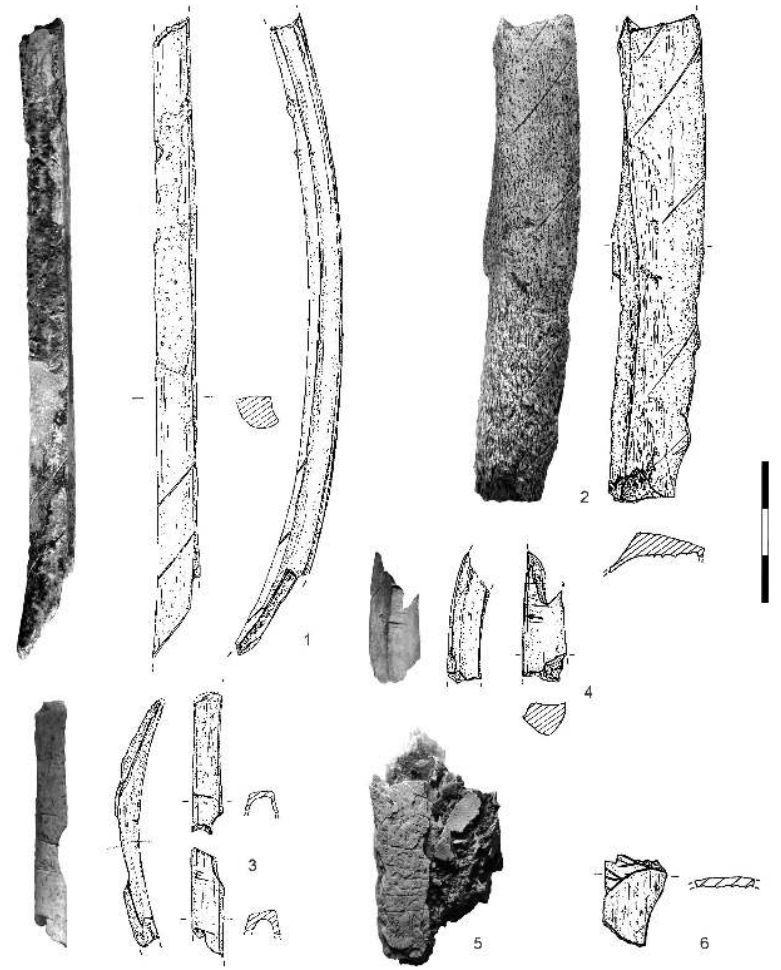

Figure 9 - Diaphyses ou fragments de côtes d'herbivores portant des traces de décarnisation ou ayant servi de billot (dessins S. Hinguant, photographie et infographie R. Colleter).

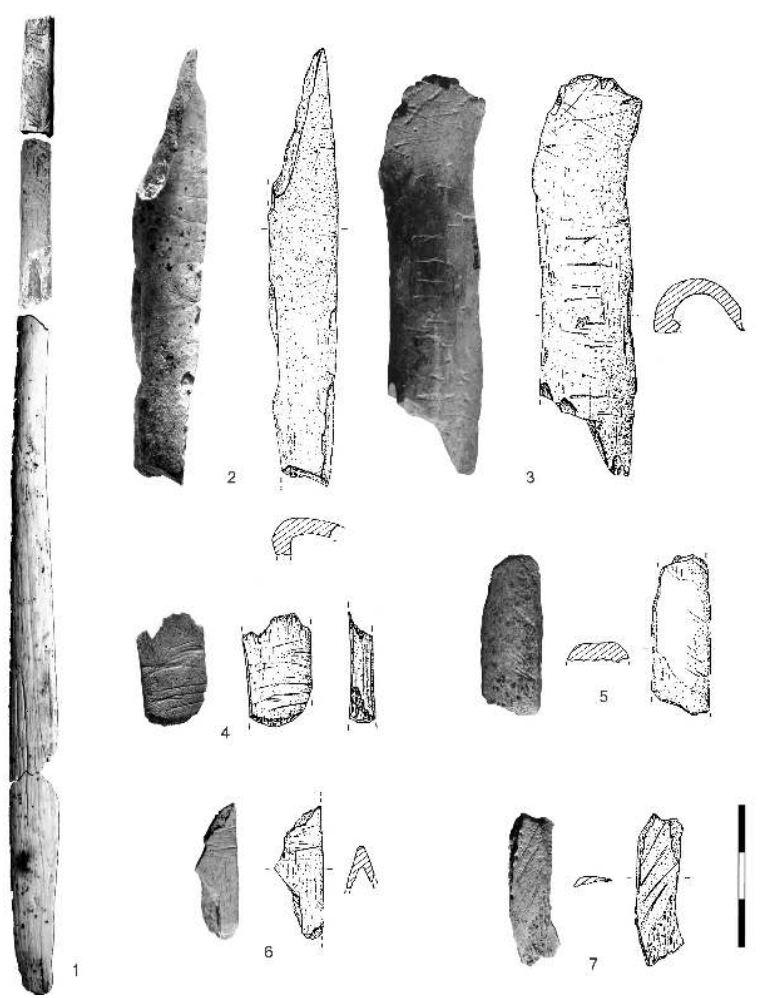




\section{4 - Une pièce particulière}

- n 6175 (4.4) : métacarpien vestigial de renne incisé (fig. 10). Sur chaque membre antérieur, les métacarpiens II et V sont des métacarpiens vestigiaux. Il s'agit ici soit d'un métacarpien $\mathrm{V}$ (bord latéral) du membre gauche, soit d'un métacarpien II (bord médial) du membre droit. Il mesure $60,7 \mathrm{~mm}$ de longueur pour $8,9 \mathrm{~mm}$ de largeur et 5,7 $\mathrm{m}$ d'épaisseur. L'extrémité apicale du métacarpien a une fracture récente.

Des traces anthropiques ont été repérées à deux endroits de l'objet. La première zone travaillée s'étend sur $7,4 \mathrm{~mm}$ et se localise, selon la position anatomique, sur l'extrémité apicale du bord droit. Elle se compose de 11 sillons et de 17 stries pour ce qui en est conservé. Les sillons ont été formés par incision qui consiste en un passage répété d'un tranchant, créant une gorge profonde. Les stries sont plus superficielles et ont été produites par un seul geste tranchant. Leur répartition par rapport aux sillons indique qu'elles pourraient être le résultat de la sortie du tranchant de son axe lors de l'incision. La seconde zone travaillée se situe, selon la position anatomique, sur la face supérieure, dans la partie mésiale du métacarpien, à $26,2 \mathrm{~mm}$ de la poulie. Elle se compose de trois stries transversales et parallèles entre elles et au plan de la pièce. Aucune trace d'utilisation macroscopique ou microscopique n'a été identifiée.

Figure 10 - Détails des sillons (en noir) et des stries (en gris) du métacarpien vestigial de renne (Rangifer tarandus) $n^{\circ} 6175$ (photo et infographie C. Peschaux).

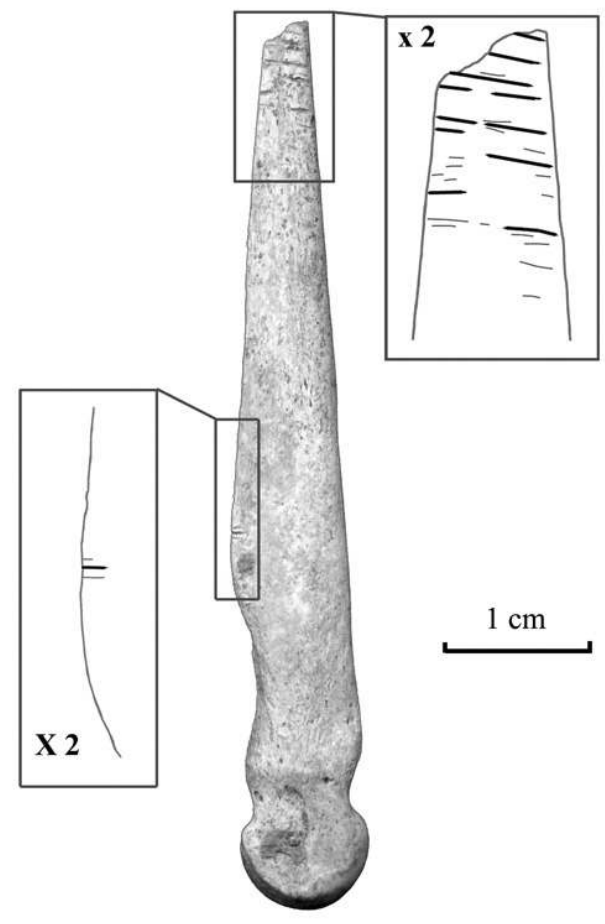

\section{3 - Éléments de comparaisons}


Tableau 1 - Inventaire récapitulatif des côtes et os gravés du Solutréen de la grotte Rochefort. La position des stries s'entend par rapport au grand axe des pièces. Le nombre de stries est un minimum, les petites incisions complémentaires, les dédoublements et les autres stries discrètes ou douteuses n'étant pas prises en compte. En grisé, pièces portant incontestablement une expression graphique.

\begin{tabular}{|c|c|c|c|c|c|c|}
\hline & Type & Espèce & $\begin{array}{c}\text { Dimensions } \\
\mathrm{mm}\end{array}$ & $\begin{array}{l}\text { Face } \\
\text { gravée }\end{array}$ & $\begin{array}{c}\text { Position stries } \\
\text { / oblique } \\
\text { | orthogonale } \\
\text { / mixte }\end{array}$ & $\begin{array}{c}\text { Nombre } \\
\text { de } \\
\text { stries }\end{array}$ \\
\hline \multicolumn{7}{|c|}{ Couche 4.2} \\
\hline 984 & côte & Ours brun & $400 \times 75 \times 20$ & interne & 1 & 14 \\
\hline 991 et rac. & côte & Bouquetin? & $185 \times 36 \times 7$ & int./ext. & il & 27 \\
\hline 1757 & métatarsien & Renne & $92 \times 15 \times 7$ & dorsale & I & - \\
\hline 1817 & côte & Renne/Équid? & $103 \times 20 \times 5$ & & 1 & 5 \\
\hline 1837 & côte & petit herb. & $43 \times 8 \times 4$ & externe & I & 6 \\
\hline 1870 & côte & Bouquetin? & $136 \times 20 \times 12$ & interne & 1 & 3 \\
\hline $\mathrm{NC} 1$ & côte & ind. & & & 1 & 7 \\
\hline NC fo 1 & côte & ind. & & & II & ind. \\
\hline \multicolumn{7}{|c|}{ Couche 4.3} \\
\hline 2189 & côte & Bouquetin? & $58 \times 11 \times 5$ & int./ext. & $\sqrt{11}$ & $8 / 3$ \\
\hline 2304 & fémur & Renne/Bouq. ? & $83 \times 23 \times 13$ & \begin{tabular}{|c|} 
dorsale \\
\end{tabular} & $1 /$ & ind. \\
\hline 2951 & cóte & Bouquetin & $111 \times 16 \times 10$ & & I & $14(15)$ \\
\hline 3167 & ind. & ind. & $50 \times 20 \times 10$ & & 1 & 9 \\
\hline 6935 & côte? & ind. & $50 \times 12 \times 5$ & externe & 1 & 11 \\
\hline 8110 & défense & Mammouth & $170 \times 35$ & \begin{tabular}{|l} 
externe \\
\end{tabular} & 1 & 11 \\
\hline $\mathrm{NC}$ do 1 & ind. & ind. & & & 1 & 3 \\
\hline NC de 1 & côte & petit herb. & & interne & 1 & 3 \\
\hline $\mathrm{NC}$ de 2 & côte & petit herb. & & interne & 1 & 2 \\
\hline $\mathrm{NC}$ de 3 & côte. & Bouquetin? & & externe & 1 & 3 \\
\hline NC de 4 & ind. & ind. & & & 1 & 4 \\
\hline $\mathrm{NC}$ de 5 & côte & petit herb. & & externe & $T$ & 2 \\
\hline \multicolumn{7}{|c|}{ Couche 4.4} \\
\hline 4268 & côte & herbivore? & $14 \times 9 \times 3$ & int.lext. & 1 & $5 / 3$ \\
\hline $5203 a$ & côte & ind. & $24 \times 15 \times 4,5$ & ind. & 1 & 6 \\
\hline 6175 & métacarpien & Renne & $60,7 \times 9 \times 5,7$ & extr. ap. & 1 & 11 \\
\hline \multicolumn{7}{|c|}{ Provenance inconnue } \\
\hline Dr. 242 & côte & petit herb. & & externe & 1 & 14 \\
\hline
\end{tabular}

8 À l'issue de cet inventaire, il est à noter que la majeure partie des pièces présentées provient des couches 4.3 (12) et 4.2 (10), montrant la validité et la relative homogénéité de la stratigraphie enregistrée (tabl. 1). L'inventaire, à l'exception notable du fragment de défense de mammouth et de l'os vestigial de renne, concerne en grande partie des côtes ou fragments de côtes et aucune des pièces n'entre dans les corpus habituels des outils ou éléments de parures. Il s'agit, pour la plupart, de côtes d'herbivores de petite ou moyenne taille, notamment le renne ou le bouquetin, ce qui pose d'emblée la question du choix des supports une fois exclues les pièces incontestablement liées à des activités de boucherie. Des côtes de grande taille, à l'instar de celle de l'ours brun, étaient disponibles et à l'exception de cette dernière, aucune ne porte de marques intentionnelles. Alors qu'il apparaît majoritaire dans le spectre faunique établi pour le site, le cheval n'est ainsi jamais mentionné (sauf peut-être la pièce 1870), espèce pourtant mise à profit dans d'autres sites, par exemple dans le Magdalénien inférieur du Taillis-des-Coteaux (Primault et al. 2010, fig. 17). Notons également qu'un seul bois de cervidé est affecté d'incisions, d'ailleurs liées au tronçonnage de la pièce $\left(\mathrm{n}^{\circ} 3237\right.$ 4.4, fig. 11), alors que de nombreux fragments d'épois ou des tronçons de bois sont bien présents dans la couche solutréenne.

9 La plupart des pièces ne semblent pas avoir subi un traitement préalable à la réalisation des incisions, quelques traces de raclage voire de poli étant néanmoins perceptibles sur certaines pièces, notamment la côte d'ours. Cependant, des lustrés taphonomiques affectent souvent les objets et il est parfois difficile de faire la part des choses entre actions naturelles et anthropiques. Aucun stigmate n'est également perceptible qui permettrait d'envisager une préparation du support en termes de dimensions. La 
plupart des pièces sont des fragments et il est difficile de savoir si les côtes ont été volontairement tronçonnées pour leur donner les dimensions recherchées. Cela ne semble pas être le cas sur les exemplaires quasi complets de la collection ( $\mathrm{n}^{\circ} 984 \mathrm{ou}$ 1870) et l'on peut supposer qu'à l'origine les décors étaient réalisés sur des côtes entières, la fragmentation pouvant être liée à un acte volontaire postérieur ou aux phénomènes post-dépositionnels après l'abandon.

Les décors sont redondants, composés de séries plus ou moins régulières de stries sur une ou plus rarement deux faces des os. Parallèles, équidistantes, orthogonales ou obliques par rapport au grand axe des pièces, ces incisions sont, dans la plupart des cas, sans lien avec de simples marques de découpe même si la prudence s'impose dans certains cas, des actions de boucherie pouvant produire ces types d'agencements (Giacobini et Patou-Mathis 2002 - p. 24, fig. 3). La position fréquente des incisions sur la face externe des côtes est cependant ici peu compatible avec ce type d'activité. Parfois fines et discrètes, les incisions peuvent aussi être plus profondément marquées, formant de véritables sillons comme dans le cas de la côte d'ours, et renforçant le caractère intentionnel des gestes.

11 Ce type de décor sur côte est fréquent dans l'art du Paléolithique supérieur notamment dans les groupes gravettiens (San Juan-Foucher 2006) et magdaléniens (Lucas 2011) mais le lot de la grotte Rochefort constitue une série pour le moins riche et originale pour le Solutréen. Si des pièces similaires ou proches sont signalées pour des sites contemporains, malheureusement souvent issues de fouilles anciennes (par exemple à Solutré, au Roc-de-Sers... cf. Combier 2002 ; Tymula 2002), les séries en question font plutôt état d'objets fonctionnels. On ne compte ainsi à Rochefort aucune armature de sagaie, aiguille, alène, aucun poinçon, lissoirs ou encore de pendeloque, pièces fonctionnelles ou ornementales parfois encochées dans le Solutréen (Le Placard, Le Fourneau-du-Diable, Pech de la Boissière... cf. Smith 1966 ; Chollot-Varagnac 1980). Dans les nombreux sites où des exemplaires sont signalés, les côtes gravées semblent davantage anecdotiques et ne représentent jamais la majorité des pièces osseuses décorées, ce qui est le cas pour la grotte Rochefort. 
Figure 11 - Épois de renne affecté dans sa partie basale par trois incisions profondes et parallèles. (n॰3237 - 4.4) (dessin S. Hinguant, photo P.-É. Moullé).
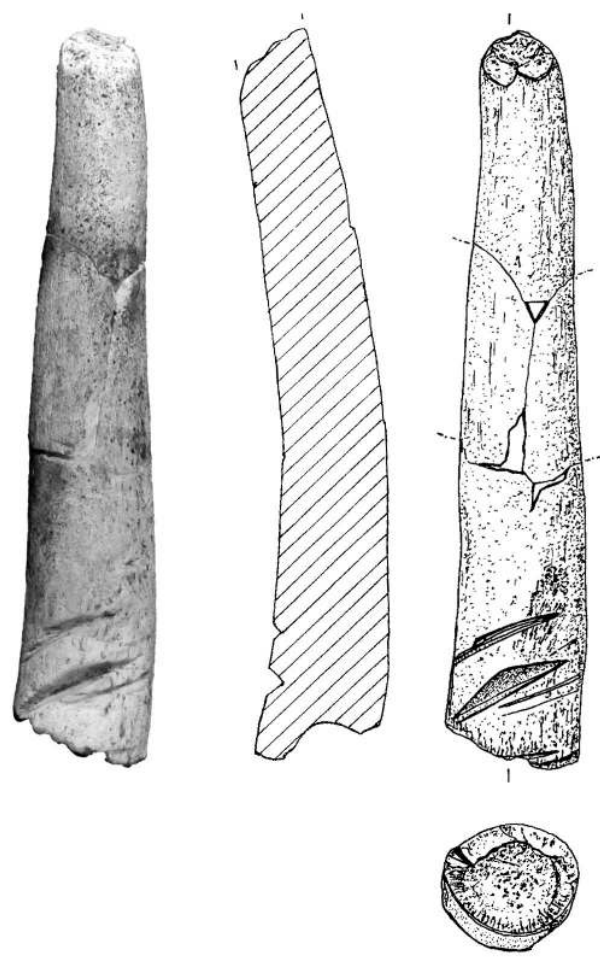

Les comparaisons s'arrêtent donc à celles entre pièces plutôt qu'entre séries. Notons déjà que les deux objets les plus remarquables de la grotte Rochefort, la côte d'ours et le fragment de défense de mammouth, ne trouvent à ce jour aucun équivalent dans les collections. On peut donc d'abord se poser la question du support choisi par le ou les artisans pour réaliser ces décors. Dans le cas de la côte d'ours, si un but fonctionnel a été recherché, peut-être faut-il évoquer les arcs miniatures ou plutôt les archets à feu ou foret confectionnés sur côtes de grands herbivores, mentionnés dans le mésolithique danois ou l'ethnographie. «La morphologie de l'objet est [alors] fortement liée à celle du support d'origine, en l'occurrence une grande côte..., long fût rectiligne ayant un profil assez courbe » (David 2001). Dans le cas présent, nous ne pouvons cependant pas certifier cette utilisation, l'absence des extrémités et donc d'éventuelles perforations ou coches de maintien d'une corde étant rédhibitoire. L'aspect localement poli de la pièce, comme les fines stries longitudinales, peuvent-ils néanmoins témoigner d'une utilisation prolongée ? L'utilisation de restes osseux d'ours dans l'art paléolithique n'est pas inédite mais elle concerne cependant souvent l'ours des cavernes, notamment des dents et griffes perforées, beaucoup plus rarement l'ours brun pour lequel les mentions concernent davantage les sites d'Europe orientale (Kozlowski 1992 ; Abramova 1995).

À notre connaissance, le fragment de défense de mammouth est à ce jour unique dans le Solutréen franco-cantabrique. L'utilisation de l'ivoire de mammouth est largement connue durant le Paléolithique supérieur, y compris au Solutréen (Castel et Madelaine 2006), que ce soit pour la confection d'outils, d'éléments de parure, d'art (les fameuses statuettes aurignaciennes et gravettiennes...) ou même, dans le Paléolithique d'Europe centrale, dans la construction d'abris. Mais aucune pièce connue ne semble supporter la comparaison avec celle de la grotte Rochefort, à l'exception peut-être du fragment d'ivoire de mammouth décoré découvert au Pech de La Boissière (Dordogne), dénommé 
« pelle rituelle » par Élie Peyrony (Peyrony 1934 ; voir également Smith 1966 - p. 192, pour une description et un dessin plus détaillés). L'objet est de taille un peu plus grande que l'exemplaire de la grotte Rochefort, le support étant sans doute prélevé sur un tronçon davantage proximal d'une défense, et ne montre pas d'incisions équivalentes mais une série de coches et striations parallèles sur les deux bords ainsi que des ponctuations en partie distale. C'est l'arrondi marqué de la partie distale et les traces d'usure et de poli qui l'affectent qui font penser à l'auteur que cet objet, semblant bien provenir du niveau solutréen de l'abri, a été utilisé comme pelle.

Si aucun élément solutréen n'est pour le moment signalé, l'utilisation de métacarpiens vestigiaux de rennes comme support de parure est connue au cours du Paléolithique supérieur. Elle a particulièrement été repérée dans les sites du Badegoulien où on en retrouve des exemplaires sciés et/ou percés dans les sites de l'abri Fritsch (Peschaux 2008) et de Cassegros (Le Tensorer 1981). Deux exemplaires sont également signalés dans le Magdalénien inférieur de Saint-Germain-la-Rivière (Vanhaeren 2002). On en retrouve également en plus grand nombre dans les collections du Placard mais dont les provenances stratigraphiques ne sont pas définies. Si le caractère volontaire des incisions semble admis, la fonction de l'exemplaire de la grotte Rochefort reste sujette à discussion. La localisation des incisions sur le bord droit, face interne non exposée, exclut l'interprétation de celles-ci comme traces de découpe. Envisagée dans un premier temps comme un possible élément de parure, cette pièce ne comporte aucun indice significatif (aménagement d'une suspension, traces d'utilisation) qui justifierait un emploi en tant que tel. Il paraît donc plus prudent de voir cette pièce comme un objet décoré, d'où sa présentation dans ce corpus des os gravés.

Le corpus fait donc état de dix pièces, auxquelles s'ajoute le métacarpien vestigial de renne, pour lesquelles les incisions sont formellement sans lien avec des traces de découpe. Six autres sont plus douteuses quant à l'intentionnalité des stries mais l'aspect de ces pièces n'évoque pas non plus strictement de simples actes de boucherie. D'une manière générale, la composition des décors est redondante, simples stries plus ou moins profondes, régulières, équidistantes et organisées de manière oblique ou orthogonale par rapport au grand axe des os. Si des rythmes sont parfois décelables ( ${ }^{\circ}$ 991) ou que les deux faces peuvent être gravées ( $n^{\circ} 2189$ et 4268), l'agencement des décors parait souvent aléatoire. C'est surtout l'impression générale de la régularité et le caractère géométrique de ceux-ci qui demeurent, faisant de ce type de composition un modèle ubiquiste, difficilement discernable, sur le plan chronoculturel, de l'ensemble des pièces recensées pour le Paléolithique supérieur. À ce titre, on ne signale pas dans la série les fréquents décors de coches ou crans serrés, profondément marqués et souvent en position latérale sur les supports, qui caractérisent les pièces solutréennes.

\section{4 - Conclusion}

Les principaux types de marques osseuses inventoriés dans la littérature et les doutes qui peuvent subsister sur l'intentionnalité de certains d'entre eux font que la prudence s'impose. M. Lorblanchet (1999) a proposé une série de critères diagnostiques pour distinguer ce qui est anthropique et ce qui ne l'est pas, ce qui est volontaire et ce qui ne l'est pas. Suivant ces critères, par la forme, le rythme et la position des incisions, et surtout compte tenu des supports utilisés, à savoir essentiellement des côtes de petits herbivores, la plupart des objets de la grotte Rochefort portent bien un décor 
volontairement tracé. Nous excluons de prime abord tout aspect fonctionnel. Il ne peut en effet s'agir d'outils ou de fragments d'outils sur lesquels des stries d'adhérence, connues par ailleurs, auraient pu être conçues pour l'emmanchement ou la fixation d'éléments connexes (Allain et Rigaud 1986). Il n'est également pas possible que les actions taphonomiques ou l'activité des rongeurs soient les seuls intervenants même si certaines traces appartiennent sans doute à ces catégories de stigmates. La décarnisation ou l'utilisation comme billot, évoquées, ne peuvent enfin à elles seules satisfaire pour interpréter l'ensemble de la collection.

Ce type d'incisions se caractérise essentiellement par leur rythme et leur répétition (Leroi-Gourhan 1965). Les espaces entre les incisions ne sont en effet pas dus au hasard et montrent une certaine régularité, composant des unités graphiques bien identifiables. Il n'est pas question ici de souligner l'émergence d'un comportement symbolique ou esthétique, phénomène déjà ancien pour l'époque dont nous parlons. Évoquons pour mémoire les vestiges très anciens de la grotte de Blombos (Afrique du sud) entre 100000 et 70000 ans (D'Errico 2003 ; D'Errico et al. 2001 ; Henshilwood et al. 2011) et de nombreux sites du Paléolithique supérieur européen (Lorblanchet 1999 ; Kozlowski et al. 1995) ou du Proche-Orient (Belfer-Cohen et Bar Yosef 2009). L'art des cavernes comporte aussi de telles marques répétitives. Que l'on songe par exemple aux traits gravés sur la corne tenue par la Vénus de Laussel (Dordogne) ou aux «peignages» de paroi récemment identifiés par M. Lorblanchet sur les parois des grottes du Quercy (Lorblanchet 2009).

Il a un temps été proposé que de telles marques impliqueraient de véritables systèmes de comptage paléolithiques (des proies chassées, des phases de la Lune, des menstrues, voir par exemple Pernat 2001 p. 49-59). Alexander Marshack (1972) est celui qui est allé le plus loin dans ces propositions. Ces hypothèses sont bien entendu invérifiables et peut-être trop simplistes (d'Errico 1996). Sur une côte de rhinocéros issue du site de Solutré, Francesco D'Errico tente de déterminer l'existence d'un système de notation à partir des 47 entailles réalisées sur la pièce (Lagardère 2006 - p. 44). Si l'organisation des incisions ne laisse guère de doute et que la technique d'entaille à l'aide d'un tranchant brut de silex est identifiée, l'auteur ne peut pas se prononcer sur la chronologie de l'exécution, excluant tout espoir de déterminer quelconque comptage.

Manifestement, certains de ces objets appartiennent donc bien à la sphère symbolique des Solutréens, ce qui ne signifie pas pour autant qu'il faille y voir l'indice d'une préoccupation esthétique, d'autres, rituelles par exemple (Pigeaud 2007), restant possibles. Insistons donc ici sur un type de comportement pour la première fois signalé dans la vallée de l'Erve et, plus largement, rarement évoqué pour le Solutréen. Trop peu d'éléments de comparaison et les provenances souvent inconnues ou peu fiables des collections anciennes de sites majeurs, Solutréens ou autres, empêchent en effet d'enrichir l'analyse (San Juan-Foucher et Vercoutère à paraître). Ce nouveau point sur la carte et dans les grilles d'inventaires est donc un jalon important pour une meilleure compréhension de l'espace culturel du Solutréen, encore mal connu.

Les données préliminaires présentées dans le cadre de cet article sont alors encourageantes. La partie fouillée de la grotte Rochefort ne correspond effectivement qu'à une surface réduite, a fortiori en périphérie de la zone d'habitat supposée. La programmation à venir devrait concerner la seconde moitié de la salle, pour laquelle nous savons qu'aucune fouille ancienne n'a affecté la stratigraphie et donc l'organisation spatiale de l'occupation solutréenne. Le corpus des pièces osseuses 
gravées devrait donc probablement s'enrichir et permettre d'affiner les recherches dans ce domaine.

Les fouilles des sites de la vallée de l'Erve bénéficient d'un financement du Conseil général de la Mayenne et du Ministère de la Culture (SRA Pays de la Loire). Les auteurs tiennent à remercier pour leur aide et conseils lors de la rédaction de cet article leurs collègues Rozenn Colleter, Cristina San Juan-Foucher, Stéphane Madelaine, Nicolas Mélard, Stéphane Péan et Caroline Peschaux. Nous remercions également vivement pour leur relecture avisée les rapporteurs de l'article, Elena Man-Estier et Valérie Feruglio. Merci enfin à Caroline Savalle pour la traduction du résumé et des légendes de figures en anglais.

\section{BIBLIOGRAPHIE}

ABRAMOVA Z.A. 1995 - L'art paléolithique d'Europe orientale et de Sibérie. Grenoble : éd. Jérôme Million, $367 \mathrm{p}$.

ALLAIN J., RIGAUD A. 1986 - Décor et fonction : quelques exemples tirés du Magdalénien. L'Anthropologie, 90 (4), p. 713-738.

ARMAND D. 2006 - Abri Castanet (Dordogne, France) : an Aurignacian Site with Bear Procurement. Bear Exploitation in Palaeolithic Time. Scientific Annals, School of Geology, Aristotle University of Thessaloniki, Special vol. 98, p. 263-268.

ARMAND D., PLASSARD F., Prat F. 2003 - L'ours des cavernes de Font-de-Gaume III. Paleo, 15, p. 241-244.

BELFER-COHEN A. et BAR-YOSEF 0. 2009 - First Things First: Abstract and Figurative Artistic Expressions in the Levant. In : P.G. Bahn (ed.), An Enquiring Mind, Studies in Honor of Alexander Marshack, Oxford : Oxbow Books, p. 25-37.

CASTEL J.-C. et MADELAINE S. 2006 - Quelques éléments remarquables de la faune du Solutréen de Laugerie-Haute (Les-Eyzies-de-Tayac, Dordogne). Paleo, 18, p. 275-284.

CHOLLOT-VARAGNAC M. 1980 - Les origines du graphisme symbolique. Essai d'analyse des écritures primitives en préhistoire. Paris : éd. Fondation Singer-Polignac, $476 \mathrm{p}$.

COMBIER J. 2002 - Témoins artistiques à Solutré. In : J. Combier et A. Montet-White (dir.), Solutré 1968-1998. Paris : Société Préhistorique Française, Mémoire XXX, p. 253-263.

DAVID É. 2001 - Fiche : arc miniature sur côte de grand ruminant. In : D. Ramseyer (dir.), Industrie de l'os préhistorique, fiches de la Commission de nomenclature sur l'industrie de l'os préhistorique, cahier IX, objets méconnus. Paris : Société Préhistorique Française, p. 91-96.

D’ERRICO F. 1996 - Marshack's approach: poor technology, biased science. Cambridge Archaeological Journal, 6 (2), p. 39-45.

D'ERRICO F. 2003 - The invisible frontier. A multiple species model for the origin of behavioral modernity.Evolutionary Anthropology, 12 (4), p. 188-202. 
D'ERRICO F, HENSHILWOOD C.S., NILSSEN P. 2001 - An engraved bone fragment from c. 70,000 year-old Middle Stone Age levels at Blombos Cave, South Africa; Implications for the origin of symbolism and language. Antiquity, 75, p. 309-318.

GIACOBINI G. et PATOU-MATHIS M. 2002 - Fiche rappels taphonomiques. In : M. Patou-Mathis (dir.), Compresseurs, percuteurs, retouchoirs, cahier X., Fiches de la Commission de nomenclature sur l'industrie de l'os préhistorique, Paris : éditions Société Préhistorique Française, p. 21-28.

HENSHILWOOD C.S, D'ERRICO F., VAN NIEKERK K., COQUINOT Y., JACOBS Z., LAURITZEN S.-E., MENU M., GARCIA-MORENO R. 2011 - A 100,000-Year-Old Ochre-Processing Workshop at Blombos Cave, South Africa.Science, 334, p. 219-222.

HINGUANT S. et BIARD M. à paraître - Le Paléolithique supérieur ancien de la vallée de l'Erve (Mayenne) : un état des connaissances. In : P. Bodu, L. Chehmana, G. Dumarçay, L. Klaric, L. Mevel, C. Peschaux, S. Soriano, N. Teyssandier (coord.), Le Paléolithique supérieur ancien de l'Europe du Nord-Ouest, Actes de la séance de la Société Préhistorique Française, Musée de Sens (Yonne), 15-18 Avril 2009.

HINGUANT S., BIARD M., MOULLÉ P.-E., PIGEAUD R. à paraître - La vallée de l'Erve (Mayenne) : présence solutréenne au nord de la Loire. In : Le Solutréen, 40 ans après Smith'66, actes du colloque international de Preuilly-sur-Claise, 28 oct.- 1er nov. 2007.

HINGUANT S., COLLETER R. (dir.) avec les contributions de ARELLANO A., BÉAREZ P., BEMILLi C., BIARD M., BODINIER B., CHAUT J.-J., DEBRET M., DESCLAUX E., EL GUENNOUNI K., HANQUET C., LAIGNEL B., LENORMAND A., MARCOUX N., MOULLÉ P.- É., NOËL F., PESCHAUX C., PIGEAUD R., POMMIER V., RODET J., ROGER T., TSOBGOU AHOUPÉ R., VISSAC C. 2010 - Le Solutréen de la grotte Rochefort (Saint-Pierre-sur-Erve, Mayenne). Rapport de fin d'opération triennale 2008-2010, campagne 2010 (3/3), Rennes et Nantes, Service régional de l'archéologie des Pays de la Loire, 210 p.

KOZLOWSKI J.K. 1992 - L'art de la préhistoire en Europe orientale. Paris : éd. CNRS, 223 p.

KOZLOWSKI J.K., SIRAKOV N., LAVILLE H., CREMADES M. 1995 - Une pierre gravée de 50000 ans B.P. dans les Balkans. Paleo, 7, p. 201-209.

LAGARDÈRE G. 2006- L'art à Solutré. In : Solutré, Musée départemental de préhistoire. Catalogue d'exposition, p. 44-45.

LE TENSORER J.-M. 1981 - Le Paléolithique de l'Agenais. Cahiers du Quaternaire, 3 (3), 528 p.

LEROI-GOURHAN A. 1965 - Le geste et la parole. Tome II, La mémoire et les rythmes.Paris : éd. Albin Michel, $288 \mathrm{p}$.

LORBLANCHET M. 1999 - La naissance de l'art. Genèse de l'art préhistorique. Paris : éd. Errance, 304 p.

LORBLANCHET M. 2009 - Claw Marks and Ritual Traces in the Paleolithic Sanctuaries of the Quercy. In : P.-G. Bahn (ed.), An Enquiring Mind, Studies in Honor of Alexander Marshack, Oxford : Oxbow Books, p. 165-170.

LUCAS C. 2011 - Gravures sur côtes : les combinaisons graphiques du Magdalénien moyen d'Isturitz (Pyrénées-Atlantiques). Paleo, 22, p. 189-206.

MARSHACK A. 1972 - The Roots of Civilization: the Cognitive Beginning of Man's First Art, Symbol and Notation. New York, ed. McGraw-Hill, 413 p.

MONNIER J.-L., HINGUANT S., PIGEAUD R., ARELLANO A., MÉLARD N., MERLE D., MOLINES N., MOULLÉ P.- É. 2005 - Art mobilier et parures sur matières dures animales : collections anciennes et découvertes récentes dans le Paléolithique supérieur de la vallée de l'Erve (Mayenne). In : V. Dujardin (coord.), industrie osseuse et parures du Solutréen au Magdalénien en Europe. Actes de la table 
ronde sur le Paléolithique supérieur récent, Angoulême, 28-30 mars 2003. Mémoire de la Société Préhistorique Française, 39, p. 101-121.

PERNAT M. 2001 - Recherches sur l'art préhistorique.Gap : Impr. Louis Jean, 171 p.

PESCHAUX C. 2008 - La question de l'homogénéité badegoulienne, apports de l'étude comparative des éléments de parure issus des niveaux badegouliens de Oisy (Nièvre) et de l'abri Fritsch (Indre). Paris : Université de Paris 1, Mémoire de Master 2, 68 p.

PEYRONY É. 1934 - Station préhistorique du Pech de la Boissière (Dordogne). Bulletin de la Société Préhistorique Française, 31 (4), p. 194-213.

PIGEAUD R. 2007 - Les rituels des grottes ornées. Rêves de préhistoriens, réalités archéologiques. In : Sophie A. de Beaune (dir.), Chasseurs-cueilleurs. Comment vivaient nos ancêtres du Paléolithique supérieur, Actes du colloque international « Restituer la vie quotidienne au Paléolithique supérieur », Lyon, 16-18 mars 2005, Paris : éd. CNRS, p. 161-170.

PIGEAUD R. à paraître. L'Ouest : carrefour ou périphérie ? Observations sur l'art pariétal et mobilier du Paléolithique supérieur ancien des grottes de Saulges. In : P. Bodu, L. Chehmana, G. Dumarçay, L. Klaric, L. Mevel, C. Peschaux, S. Soriano, N. Teyssandier (coord.), Le Paléolithique supérieur ancien de l'Europe du Nord-Ouest, Actes de la séance de la Société Préhistorique Française, Musée de Sens (Yonne), 15-18 Avril 2009.

POPLIN F. 1995 - Délitage et débitage dans le travail de l'ivoire vrai sur des exemples du début du Paléolithique supérieur. In : J. Hahn, M. Menu, Y. Taborin, P. Walter, F. Windermann (éd.), Le travail et l'usage de l'ivoire au Paléolithique supérieur. Actes de la table ronde de Ravello, 29-31 mai 1992. Rome : Istituto Poligrafico e Zecca dello Stato, Libreria dello Stato, p. 17-27.

PRIMAULT J., avec la collaboration de BERTHET A.-L., BROU L., DELFOUR G., GABILLEAU J., GRIGGO C., GUÉRIN S., HENRY-GAMBIER D., HOUMARD C., JEANNET M., LACRAMPE-CUYAUBÈRE F., LANGLAIS M., LAROULANDIE V., LIARD M., LOLIOS D., LOMPRÉ A., LUCQUIN A., MISTROT V., RAMBAUD D., SCHMITT A., SOLER L., TABORIN Y., VISSAC C. 2010 - La grotte du Taillis-desCoteaux à Antigny (Vienne). In : J. Buisson-Catil et J. Primault (dir.), Préhistoire entre Vienne et Charente, Hommes et sociétés du Paléolithique. Chauvigny : Association des Publications Chauvinoises, mémoire XXXVIII, p. 271-293.

QUILÈS J. 2004 - Tanières d'ours des cavernes (Carnivora, Ursidae) du pourtour méditerranéen : étude taphonomique et paléobiologique de huit assemblages du Pléistocène supérieur, Paleo, 16, p. 171-192.

SAN JUAN-FOUCHER C. 2006- Industrie osseuse décorée du Gravettien des Pyrénées. Munibe,Homenaje a Jesus Altuna, San Sebastian, 57 (3), p. 95-111.

SAN JUAN-FOUCHER C., VERCOUTÈRE C. à paraître - Fiche 8 ; Côtes. In : L. Mons, S. Péan et R. Pigeaud (dir.), Matière d'art : représentations préhistoriques et supports osseux, relations et contraintes.Commission de nomenclature sur l'industrie de l'os préhistorique, cahier XIII.

SMITH P.E.L. 1966 - Le Solutréen en France.Bordeaux : Publication de l'Institut de préhistoire de l'Université de Bordeaux, 5, impr. Delmas, 449 p.

TYMULA S. 2002 - L'art solutréen du Roc de Sers (Charente). Paris : éd. Maison des Sciences de l'Homme (Documents d'Archéologie Française 91), 288 p.

VANHAEREN M. 2002 - Les fonctions de la parure au Paléolithique supérieur : de l'individu à l'unité culturelle. Bordeaux : Université de Bordeaux 1, Thèse de Doctorat, 365 p. 
VERCOUTÈRE C., SAN JUAN-FOUCHER C., FOUCHER P. 2006 - Human Modifications on Cave Bear Bones from the Gargas Cave (Hautes-Pyrénées, France). Scientific Annals, School of Geology, Aristotle University of Thessaloniki, Special vol. 98, p. 257-261.

\section{RÉSUMÉS}

Au sein du corpus mobilier solutréen provenant de la grotte Rochefort, un lot de 25 pièces a été isolé. Il s'agit essentiellement de côtes d'herbivores mais aussi de fragments d'os longs et d'ivoire, présentant des incisions plus ou moins profondes pour lesquelles la disposition n'évoque pas de simples stries liées à la découpe. Des objets remarquables, comme une côte d'ours brun complète ou un fragment de défense de mammouth, font partie de cette série de pièces incisées. Symboliques, ces décors gravés sont connus dans le Solutréen mais les contextes chronostratigraphiques imprécis de la plupart des collections anciennes en font des pièces de référence peu fiables. La recherche de comparaisons portant peu ses fruits, le corpus de la grotte Rochefort se singularise donc par le nombre et la qualité des objets inventoriés.

Within the Solutrean portable corpus gathered from Rochefort cave, a series of approximately thirty items has been isolated. It is mainly made up of herbivore ribs but also of fragments of long bones and dentin which are showing some incisions with different degrees of depth, the disposition of which does not remind of simple grooves related to cutting activities. Amazing items such as an entire brown bear rib or a fragment of mammoth tusk are part of this series of incised pieces. These symbolic engraved decorations are known for the Solutrean period but the blurry chronostratigraphic contexts of most of the older collections make them unreliable items of reference. The search for comparisons has not been really fruitful, thus the Rochefort cave corpus distinguishes itself by the quantity and quality of the objects that have been listed.

\section{INDEX}

Mots-clés : Solutréen, os gravé, mammouth, ours brun, grotte Rochefort, Mayenne

Keywords : Solutrean, Engraved Bones, Mammoth, Brown Bear, Rochefort Cave, Mayenne

\section{AUTEURS}

\section{STÉPHAN HINGUANT}

INRAP-Bretagne, 37 rue du Bignon, 35577 Cesson-Sévigné, France et UMR 6566 CNRS CReAAH « Centre de Recherche en Archéologie Archéosciences Histoire », CNRS, Université de Rennes 1, Rennes 2, Nantes et Ministère de la Culture ; Bâtiment 24-25, Université Rennes 1 - Campus de Beaulieu 74205CS, 35042 Rennes Cx, France - stephan.hinguant@inrap.fr

\section{PIERRE-ELIE MOULLÉ}

Musée de Préhistoire régionale de Menton, rue Lorédan Larchey, 06500 Menton et Département de Préhistoire du Muséum National d'Histoire Naturelle, Institut de Paléontologie Humaine, Paris, France - pierre-elie.moulle@ville-menton.fr 


\section{ALMUDENA ARELLANO}

Musée de Préhistoire régionale de Menton, rue Lorédan Larchey, 06500 Menton, France almudena.arellanoalonso@ville-menton.fr

ROMAIN PIGEAUD

UMR 7194 CNRS, Département de Préhistoire du Muséum national d'Histoire naturelle, Institut de Paléontologie Humaine, Paris, France - romain.pigeaud@wanadoo.fr 